\title{
Hypoxia influences linearly patterned programmed cell necrosis and tumor blood supply patterns formation in melanoma
}

\author{
Shiwu Zhang ${ }^{1,2, *}$, Man $\mathrm{Li}^{1,3, *}$, Danfang Zhang ${ }^{1}$, Shaoyan Xu ${ }^{1}$, Xiaoyu Wang ${ }^{1}$, Zhiyong Liu ${ }^{1}$, Xiulan Zhao ${ }^{4}$ and \\ Baocun Sun ${ }^{1,4, *}$
}

To investigate the possibility that tumor cells undergoing linearly patterned programmed cell necrosis (LPPCN) establish a spatial foundation for vasculogenic mimicry (VM) and to reveal that hypoxia influences LPPCN formation as well as Endo $\mathrm{G}$ and DNase 1 expression, 78 C57 mice were divided evenly into two groups and engrafted with B16 melanoma. Starting 9 days after inoculation, subgroups of mice were killed every 2 days. LPPCN and the tumor blood supply vessel types were counted and Endo G and DNase 1 mRNA expression were measured. Additionally, 124 cases of human melanoma samples were collected to assess the clinical significance of LPPCN and VM. The data revealed that regions of LPPCN were positive for caspase-3, caspase-9 and Bax, and negative for TUNEL staining. Electron microscopy images indicated that these cells took on the morphologic changes of necrosis. There was more DNase I mRNA expression in the hypoxic group than in the control group $(P<0.05)$ in vitro, and the expression of Endo $G$ mRNA in the hypoxic groups was significantly higher than that in the control groups both in vitro and in vivo $(P<0.05)$. VM and LPPCN cell numbers in the ischemic group were higher than those in the control group in the early stage of tumor growth. Finally, the survival time for patients whose samples showed LPPCN and VM was significantly shorter than that of patients with one or neither of those factors. We speculated that under hypoxic conditions, some melanoma cells might undergo LPPCN, thus providing a spatial foundation for VM channel formation.

Laboratory Investigation (2009) 89, 575-586; doi:10.1038/labinvest.2009.20; published online 16 March 2009

KEYWORDS: melanoma; linearly patterned programmed cell necrosis; vasculogenic mimicry; endonuclease G; angiogenesis

Melanoma is a high-grade, poorly differentiated malignant tumor. The biological behavior of melanoma is characterized by rapid growth and a high metastasis rate via vessels. Rapid growth makes melanoma cells in urgent need of oxygen and nutrients supply. In the early stage of rapid growth, blood supply cannot supply sufficient oxygen and nutrition solely through angiogenesis because enough new vessels have not yet been formed. Nevertheless, angiogenesis is not the only way to supply blood to the tumor. ${ }^{1}$ New patterns other than angiogenesis have been found in some malignant tumors. Vasculogenic mimicry $(\mathrm{VM}),{ }^{2,3}$ for example, composed strictly of tumor cells without endothelial cells, is the dominant form of vessel in the early stage of some subpopulations of highly aggressive and metastatic malignant tumors. The basis for VM formation and the mechanisms involved in this process are still not clear.

Earlier, we showed that tumor cells became more invasive and formed more VM channels under hypoxic conditions, and there were some tumor cells that were darkly stained in the H\&E-stained sections that were distributed in patterns of lines and networks. ${ }^{4}$ This distribution is similar to that seen in VM networks and endothelium-dependent vessels (EV). We coined the term 'linearly patterned programmed cell necrosis' (LPPCN) to describe these darkly stained cells with special distribution patterns. In this study, we discuss the relationship between LPPCN and VM in melanoma. Programmed necrosis has recently been described by Zong et $a l^{5}$ as a controlled cell death process. This suggestion differs

\footnotetext{
Department of Pathology, Tianjin Cancer Hospital and Institute, Tianjin Medical University, Tianjin, PR China; ${ }^{2}$ Department of Pathology, Tianjin Dongli Hospital, Tianjin, PR China; ${ }^{3}$ Department of Digestive, Tianjin Second Hopital of Tianjin Medical University, Tianjin, PR China and ${ }^{4}$ Department of Pathology, Tianjin Medical University, Tianjin, PR China

Correspondence: Dr B Sun, PhD, Department of Pathology, Tianjin Cancer Hospital, Tianjin Medical University, Tianjin 300060, PR China.

E-mail: zhangshiwu666@yahoo.com.cn

*These authors contributed equally to this work.

Received 24 November 2008; revised 20 January 2009; accepted 3 February 2009
} 
from the current view of necrosis as accidental in that programmed necrosis is far from being a passive event. In fact, it involves components of orderly processes required for the activation of a cell death program. Numerous physiological and pathological processes are involved in this kind of special programmed cell death, which can be activated by many factors including tissue hypoxia, drugs and toxins. ${ }^{6,7}$ Our LPPCN model suggests that the networks of programmed cell necrosis that we have observed are the beginning of a stepwise process. In the early rapid growth stage of tumor development, oxygen and nutrition from EV are not enough for melanoma growth. ${ }^{8}$ Later, under the regulation of apoptosisrelated gene stimulation in the local hypoxic environment, LPPCN might be initiated, which eventually results in a channel-shaped vessel-like empty space left by the dead cells. Results from immunohistochemical staining (IHC) and terminal TdT-mediated dUTP-biotin nick end labeling (TUNEL) staining suggest that these cells are undergoing programmed necrosis. Thus, the empty region formed by LPPCN might be the spatial basis for VM channel formation. Moreover, VM channels could connect with EV, and when these channels and vessels meet, oxygen and nutrient-rich blood could reach an increasing number of tumor cells. The distribution and morphologic changes of LPPCN are different from apoptosis. LPPCN cells form a line shape and apoptosis appear in randomly distributed cells. Morphologically, LPPCN cells display pyknosis, karyorrhexis or karyolysis, features that are different from the apoptotic bodies and DNA fragmentation typical of apoptosis. The molecular mechanism regulating LPPCN is different form typical necrosis.

\section{MATERIALS AND METHODS Animals}

Seventy-eight 6- to 8-week-old female C57/6J black mice were purchased from the Animal Base of the Union Drug Institute (Beijing). The weight range of these mice was $23-25 \mathrm{~g}$. The mice were divided into the ischemic group and the control group with 39 mice in each group. They were further divided into five subgroups as the ninth day (nine mice), the eleventh day (nine mice), the thirteenth day (seven mice), the fifteenth day (five mice) and the seventeenth day (nine mice) subgroups.

\section{Cell Line}

Single cell suspension of the malignant melanoma cell line B16 was provided by the Department of Biochemistry in Tianjin Cancer Hospital. Before injection, the suspension was placed in a $43^{\circ} \mathrm{C}$ water bath for $20-30 \mathrm{~s}$ and then the cells were pelleted by centrifugation at $1000 \mathrm{~g}$ for $10 \mathrm{~min}$. The supernatant was removed by absorption into a sterile cotton pad and the cell pellet was resuspended with $1-2 \mathrm{ml} 0.9 \%$ $\mathrm{NaCl}$ solution to a cell density of $1 \times 10^{7}$ cells $/ \mathrm{ml}$.

\section{Ischemic Animal Model}

All 78 mice were inoculated with single cell suspensions of malignant melanoma B16 cells according to the following procedure: the mouse groin was sanitized with an alcohol swab and $0.2 \mathrm{ml}$ single tumor cell suspension was injected subcutaneously. Ischemia was produced in the left hindlimb by surgical procedure 3 days later. Under anesthesia, the mice were well restrained, and a $1-1.5 \mathrm{~cm}$ incision was made in the skin in the left groin. The femoral artery was then ligated twice with 6-0 silk and transected in two places distal to the ligature. Any other large blood vessels distal to the ligature that were visible were also transected. The wound was closed with 4-0 silk, and the hindlimbs were gently immobilized. Nine days after inoculation, tumor mass (approximate diameter of 1-2 mm) was observed in the mice at the site of injection. From the ninth to the seventeenth day one group of mice was killed every 2 days.

\section{Quantitation of VM Channels, EV and LPPCN Cells}

VM channels and EV in the H\&E stained sections were counted using $\times 400$ magnification. Five fields were chosen randomly, and the average blood supply pattern was defined as the number of microvessels or channels in one section. LPPCN cells were counted in the same field. All these counts were blindly performed in at least three randomly chosen sections from each mouse. The mean value of each type of microvessel in five fields was the final outcome.

\section{IHC Staining and Histochemistry Staining}

Four-micrometer-thick sections of formalin-fixed, paraffinembedded engrafted animal melanoma tissues were mounted on poly-L-lysine-coated slides. Slides were deparaffinized in xylene. Endo Genous peroxidase activity was blocked with $3 \%$ hydrogen peroxide in $50 \%$ methanol for $10 \mathrm{~min}$ at room temperature. Sections were rehydrated in alcohol, washed with phosphate-buffered saline (PBS) and then pretreated with citrate buffer $(0.01 \mathrm{M}$ citric acid, $\mathrm{pH} 6.0)$ for $20 \mathrm{~min}$ at $95^{\circ} \mathrm{C}$ in a microwave oven. After blocking of nonspecific binding sites by exposing them to $10 \%$ normal goat serum in PBS for $20 \mathrm{~min}$ at $37^{\circ} \mathrm{C}$, sections were incubated overnight at $4{ }^{\circ} \mathrm{C}$ with rabbit polyclonal anti-CD34 (Sigma Chemical Co., St Louis, Mo, USA), rabbit polyclonal anti-caspase-3 (Newmarker, New York, USA; dilution 1:100), rabbit polyclonal anti-caspase-9 (Newmarker; dilution 1:100), rabbit polyclonal anti-FasL (Zhongshan, Beijing, China, ready-to-use), rabbit polyclonal anti-Bax (Newmarker; dilution 1:200), rabbit polyclonal anti-caspase-8 (clone:BA0689; Boster Biological Technology Ltd, Wuhan, China; dilution 1:100), rabbit polyclonal anti-Bcl-2 (clone:BA0412; Boster Biological Technology Ltd; dilution 1:150), rabbit polyclonal anti-TNF $\alpha$ (clone:BA0131; Boster Biological Technology Ltd; dilution 1:150), rabbit polyclonal anti-TRAIL (clone:BA1446; Boster Biological Technology Ltd; dilution 1:100), rabbit polyclonal anti-caspase-7 (clone:BA0688; Boster Biological Technology Ltd; dilution 1:100), rabbit polyclonal anti-FAS 
(clone:BA0048; Boster Biological Technology Ltd; dilution 1:150) and rabbit polyclonal anti-Endo G (Chemicon International, Temecula, CA, USA; dilution 1:100). Following incubation, the sections were rinsed with PBS, incubated with biotinylated goat anti-mouse IgG for $20 \mathrm{~min}$ at $37^{\circ} \mathrm{C}$, incubated with $3,3^{\prime}$-diaminobenzidine (DAB) chromogen for 5-10 min at room temperature and then washed with distilled water. After IHC staining for CD34, the sections were then washed with running water for $5 \mathrm{~min}$ and incubated with periodic acid Schiff (PAS) for $15 \mathrm{~min}$. Finally, all of the sections were counterstained with hematoxylin, dehydrated and mounted. Normal human stomach mucous membrane was the positive control for PAS staining.

\section{TUNEL Staining}

For in situ visualization of apoptotic cell distribution, we applied the TUNEL technique. Immediately after deparaffinization and rehydration, Endo Genous peroxidase was inactivated by covering sections with $3 \%$ hydrogen peroxide for $10 \mathrm{~min}$ at room temperature. Nuclei of tissue sections were stripped of proteins by incubation with $20 \mu \mathrm{g} / \mathrm{ml}$ proteinase $\mathrm{K}$ for $10 \mathrm{~min}$ at $37^{\circ} \mathrm{C}$. Sections were covered with $\mathrm{TdT}$ and biotin-d-UTP in TdT buffer and the slides were incubated in a humid atmosphere at $37^{\circ} \mathrm{C}$ for $90 \mathrm{~min}$. The reaction was terminated by transferring the slides three times to $0.1 \mathrm{M}$ PBS buffer for $2 \mathrm{~min}$. The sections were then covered with blocking solution for $30 \mathrm{~min}$ at room temperature. Sections were incubated with biotin-labeled anti-DIG antibody diluted 1:100 in blocking solution. After washing the sections, they were then covered with streptavidin-biotin complex (SABC) diluted 1:100 in PBS and incubated at $37^{\circ} \mathrm{C}$ for $60 \mathrm{~min}$. The sections were stained with $\mathrm{DAB}$ for $7-8 \mathrm{~min}$ at room temperature. Positively labeled brown cell nuclei were visible after exposure to DAB. Positive control experiments demonstrating DNA fragmentation were performed by exposing tissue sections to deoxyribonuclease (DNase), whereas for negative controls the TdT/dUTP labeling mixture was omitted, resulting in no staining. Finally, all sections were counterstained with hematoxylin. Sections were rinsed with PBS after each step.

\section{In Situ Hybridization of Endo G}

In situ hybridization (ISH) of Endo G was performed using a commercially available kit (Boster Biological Technology Ltd.; part number NO. MK2795-m). The kit used three probes complementary to Endo G: $5^{\prime}$-AACCACCGCTGG AGTCAGCGTGGACGACAC-3' ${ }^{\prime}, 5^{\prime}$-AACCAGAATGCCTGGA ACAACCTTGAGAGGTACAG- $3^{\prime}$ and $5^{\prime}$-ATCGAGCTACGTT CCTACGTGATGCCCAATGCCCC- $3^{\prime}$. The three probes were used separately and did not match any known sequence in GenBank. ISH was carried out following the procedure described by Xu et al. ${ }^{9}$ Briefly, the sections were deparaffinized, rehydrated and deproteinized. The slides were then prehybridized at $40^{\circ} \mathrm{C}$ for $2 \mathrm{~h}$ and incubated with hybridization solution $(20 \mu \mathrm{l} /$ slide $)$ containing digoxigenin-labeled oligo- nucleotide probe at $40^{\circ} \mathrm{C}$ for $16 \mathrm{~h}$. The sections were washed twice in $2 \times$ SCC for $10 \mathrm{~min}$ and further washed in $0.5 \times$ SCC and $0.2 \times$ SCC for $30 \mathrm{~min}$. Sections were incubated with biotin-labeled anti-DIG antibody diluted at $37^{\circ} \mathrm{C}$ for $1 \mathrm{~h}$. After washing the sections with PBS, they were then covered with $\mathrm{SABC}$ and incubated at $37^{\circ} \mathrm{C}$ for $20 \mathrm{~min}$. Sections were stained with $\mathrm{DAB}$ for $5 \mathrm{~min}$ at room temperature. Finally, sections were lightly counterstained with hematoxylin for $1 \mathrm{~min}$. The specificity of the method was confirmed by the positive results obtained with corresponding sense controls and the distinct positive staining patterns obtained with digoxigenin-labeled GAPDH mRNA antisense probes (Boster Biological Technology Ltd.).

\section{Electron Microscopy}

Tumor specimens in the early and late growth stages were randomly selected and fixed with glutaraldehyde. The areas containing linearly patterned programmed necrosis were located using a light microscope. Ultra-thin sections were examined using a JEM-1010 electron microscope. Pyknosis and karyorrhexis of the nuclear chromatin were the criteria for determining cells, which were undergoing programmed necrosis.

\section{Cell Culture}

Melanoma cells were cultured with RPMI 1640 and 10\% fetal bovine serum. When cells cultured under normal conditions with $10 \% \mathrm{O}_{2}, 85 \% \mathrm{~N}_{2}$ and $5 \% \mathrm{CO}_{2}$ became $70 \%$ confluent, they were transferred to hypoxic conditions with $2 \% \mathrm{O}_{2}, 93 \%$ $\mathrm{N}_{2}$ and $5 \% \mathrm{CO}_{2}$. Melanoma cells were collected at 2, 4, 6, 8 and $24 \mathrm{~h}$. This test was performed in triplicate with tumor cells cultured under normal conditions serving as the control.

\section{Total RNA Isolation and Real-Time PCR}

Total RNA was isolated from frozen samples and melanoma cells using TRIzol reagent. The OD260/280 ratio for all samples was 1.9-2.0. cDNA was synthesized through reverse transcription. Primers were designed and synthesized by TaKaRa Biotechnology Co. Ltd. Real-time PCR analysis was carried out to determine the level of Endo G and DNase I mRNA using the Gene AMP PCR System 5700 Sequence Detector. The cDNA was used as the template to be amplified in a $25-\mu \mathrm{l}$ reaction mixture using the following conditions: denaturation at $94^{\circ} \mathrm{C}$ for $5 \mathrm{~min}$, then 35 cycles of $94^{\circ} \mathrm{C}$ for $30 \mathrm{~s}$, the optimal annealing temperature for $45 \mathrm{~s}$ and $72^{\circ} \mathrm{C}$ for $40 \mathrm{~s}$. The primer sequences used for matrix metalloproteinase Endo G (Accession No.: NM009524) detection were 5'-ACC GCATTTCTACTCGGATG-3' (sense) and 5'-CCTCCTCGGT CAGAAATCTT-3' (antisense). The primer sequences used for DNase 1 (Accession No.:NM009525) detection were $5^{\prime}$-CTGGCCCTGTCCCTGAGAATAGCA-3' (sense) and $5^{\prime}$-CTGGGTGGAGGGGGAGGAGAACT- $3^{\prime}$ (antisense). The primers used to amplify $\beta$-actin were $5^{\prime}$-CATCCGTAAAG ACCTCTATGCCAAC-3' (sense) and 5'-ATGGAGCCACCGA TCCACA- $3^{\prime}$ (antisense). The resultant products of Endo G, 
DNase 1 and $\beta$-actin amplification were 190, 184 and 174 base pairs, respectively. The $C_{\mathrm{T}}$ value (the cycle number at which the fluorescence crosses the threshold) was determined and the formula $2 \wedge(-$ deltadeltaCt $)$ was used to determine the relative quantity of the amplified fragment. Every sample was tested in triplicate and the mean value was used.

\section{Patient Samples}

Paraffin-embedded melanoma tissue samples (total 124 cases-67 male and 57 female) came from patients who underwent surgical resection in TianJin Cancer Hospital between 5 January 1984 and 20 December 2000. All of the patients died from melanoma-related causes with an average survival time of $76.29 \pm 11.51$ months.

\section{Statistics}

The data in this study were analyzed with SPSS 11.5 , and a $P$ value of 0.05 was defined as significance. One-way ANOVA was used to compare the differences in Endo G and DNase 1 mRNA expression. Kruskal-Wallis $\mathrm{H}$ test was used to detect the differences among the groups of animals killed on different days. Wilcoxon test was performed to compare the ischemic and control groups. Mann-Whitney test was used to analyze the time-dependent change of microvessel density in the ischemic and control groups. Kaplan-Meier survival analysis was used. Data from those patients who were still alive by the end of 30 December 2007 were censored.

\section{RESULTS}

\section{Microscopic and Visual Observation of Morphology}

Seven to eight days after inoculation, the tumors could be palpated and were determined to be approximately 0.2 $0.3 \mathrm{~cm}$ in diameter. Black tumor tissue invaded surrounding normal tissue with a blurred borderline (Figure 1a). After H\&E staining of sections, there were more tumor cells with condensed chromatin (LPPCN cells) appearing in the center of the tumor mass in the ischemic group. In the control group, LPPCN cells also appeared in the center of large tumor mass, but the quantity of LPPCN cells was less than that in the ischemic group. Under low magnification, these cells appear to link with each other and form a network (Figure 1b), which is LPPCN. VM channels or EV and the cells with condensed chromatin seem to colocalize. Tumor cells, some with dark nuclei that are apparently dying, can also be observed around VM channels and EV. These cells with dark nuclei can form lacunae, which may be the infrastructure of VM (Figure 1c and d). Results of CD34 and PAS double staining show that some cells with darkly stained nuclei appear around VM channels with PAS-positive substances in the basement membrane. $\mathrm{EV}$ with endothelial cells appearing brown after IHC staining for CD 34 coexisted with VM (Figure 1e) in the same field. In short, tumor cells with darkly stained nuclei not only appear in the center of large tumor masses but also colocalize with VM and EV.
In a related project, ${ }^{10}$ several B16-inoculated mice bearing melanoma of approximately 0.3 micrometers in diameter were injected with activated carbon via the tail vein, and were killed $15 \mathrm{~min}$ after the carbon injection. Many activated carbon granules were observed in the center part of channel structures formed by the cells with dark nuclei, indicating that the channels were functional for microcirculation. Activated carbon was also identified in the middle of the EV and VM channels (Figure 1f). Therefore, it appears the VM channel provides functional microcirculation with blood flowing through it to satisfy the oxygen and nutritional needs of the tumor cells.

\section{Quantitation of LPPCN, VM Channels and EV in Different Groups of Mice}

To identify the type of tumor blood supply vessels in different melanoma growth stages, we determined the density of each vessel type every other day starting on the ninth day after B16 melanoma inoculation. Although at the early stage characterized by tumor rapid growth of melanoma formation, blood supply vessel types for the tumors consisted of both $\mathrm{VM}$ and $\mathrm{EV}, \mathrm{VM}$ channels were predominant in the hypoxic group (Figure 2a and b). Moreover, VM density decreased significantly during tumor growth, whereas EV did not (Figure 2b). On the ninth day after B16 cell inoculation, tumors in the ischemic group showed more VM channels and LPPCN cells than those in the control group $(Z=-2.03$, $-1.92 ; P=0.038,0.067)$. Furthermore, in the ischemic group, LPPCN cell number was significantly higher on the ninth day than on the later days $(\mathrm{Z}=-2.93,-2.33,-1.98$, $-2.87 ; P=0.003,0.02,0.047,0.004)$, and the same trend held for $\mathrm{VM}$ channels $(\mathrm{Z}=-3.32,-2.00,-2.58,-3.18$; $P=0.001,0.042,0.016,0.001)$. Although melanomas in the control group had more VM channels and LPPCN cells on the ninth day than in the following days, the rate of decrease was much lower when compared to the number of VM channels and LPPCN cells in the ischemic group (Table 1). Presumably, this observation is due to the fact that VM channel formation was only favored under hypoxic conditions at the early stage (Figure $2 \mathrm{a}$ and $\mathrm{c}$ ).

\section{Results of IHC Staining and ISH}

Putative cell death pathways, including programmed necrosis and apoptosis, are regulated by caspase-3, caspase- 9 , Bax and FasL. ${ }^{5}$ Using IHC staining for these proteins, abundant expression (brown color) for caspase-3, caspase- 9 and Bax were detected in the plasma of the tumor cells undergoing linearly patterned programmed necrosis. These LPPCN cells were negative for TNF, TRAIL, Fas, FasL, Bcl-2 and Caspase- 8 . The distribution of cells undergoing programmed necrosis was linear and showed a trend of connecting the channels with each other. The spatial infrastructure derived from LPPCN also seems to connect with EV. Tumor cells with darkly stained nuclei lining the wall of VM and EV were positive for Bax IHC staining (Figure $3 a-c$ ). Furthermore, our results 

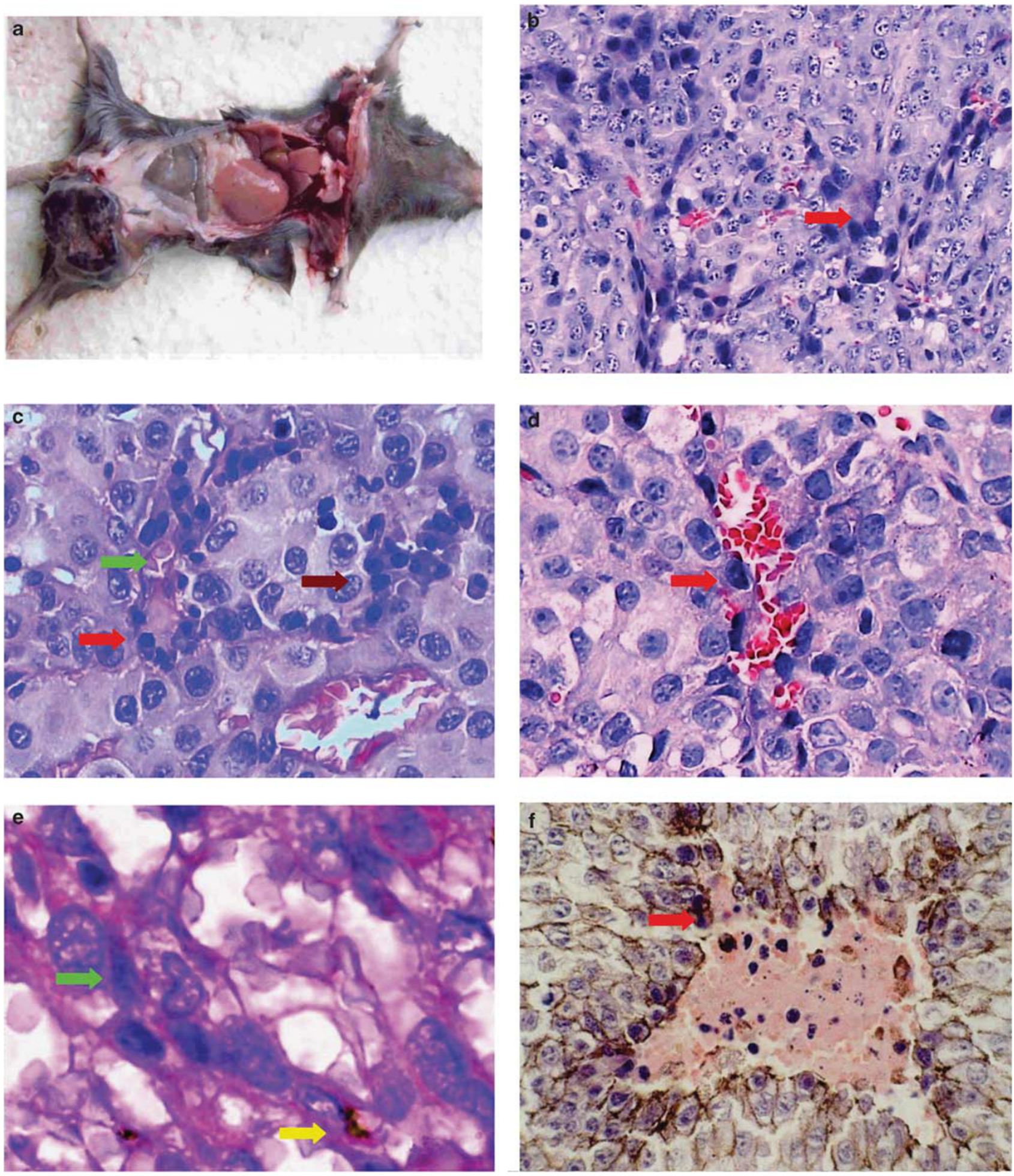

Figure 1 (a) The mouse was inoculated with B16 melanoma cell line in the groin. (b) There were more channels lined with tumor cells that showed darkly stained nuclei (red arrow) distributed in the center of the tumor tissue in the ischemic group. H\&E, $\times 400$. (c) Tumor cells with darkly stained nuclei (red arrow) distributing around EV (green arrow) suggest that there was colocalization of cells undergoing LPPCN and EV. Some LPPCN cells forming a vessel-like empty space showed a trend of connecting with EV (dark-red arrow). H\&E, $\times 400$. (d) Some tumor cells (red arrow) lining the wall of the VM channel had darkly stained nuclei. H\&E, $\times 400$. (e) A VM channel is formed by tumor cells with and without undergoing LPPCN (green arrow). There are red cells in the center of the channels. PAS-positive substances line the VM channel. Yellow arrow indicates EV in the same field with VM. Endothelial cells are stained brown by IHC staining for CD34. CD34 and PAS double staining, $\times 400$. (f) Active carbon appeared in the middle of VM channels and tumor cells undergoing programmed necrosis line the inner wall of the channel (red arrow). H\&E, $\times 400$. 

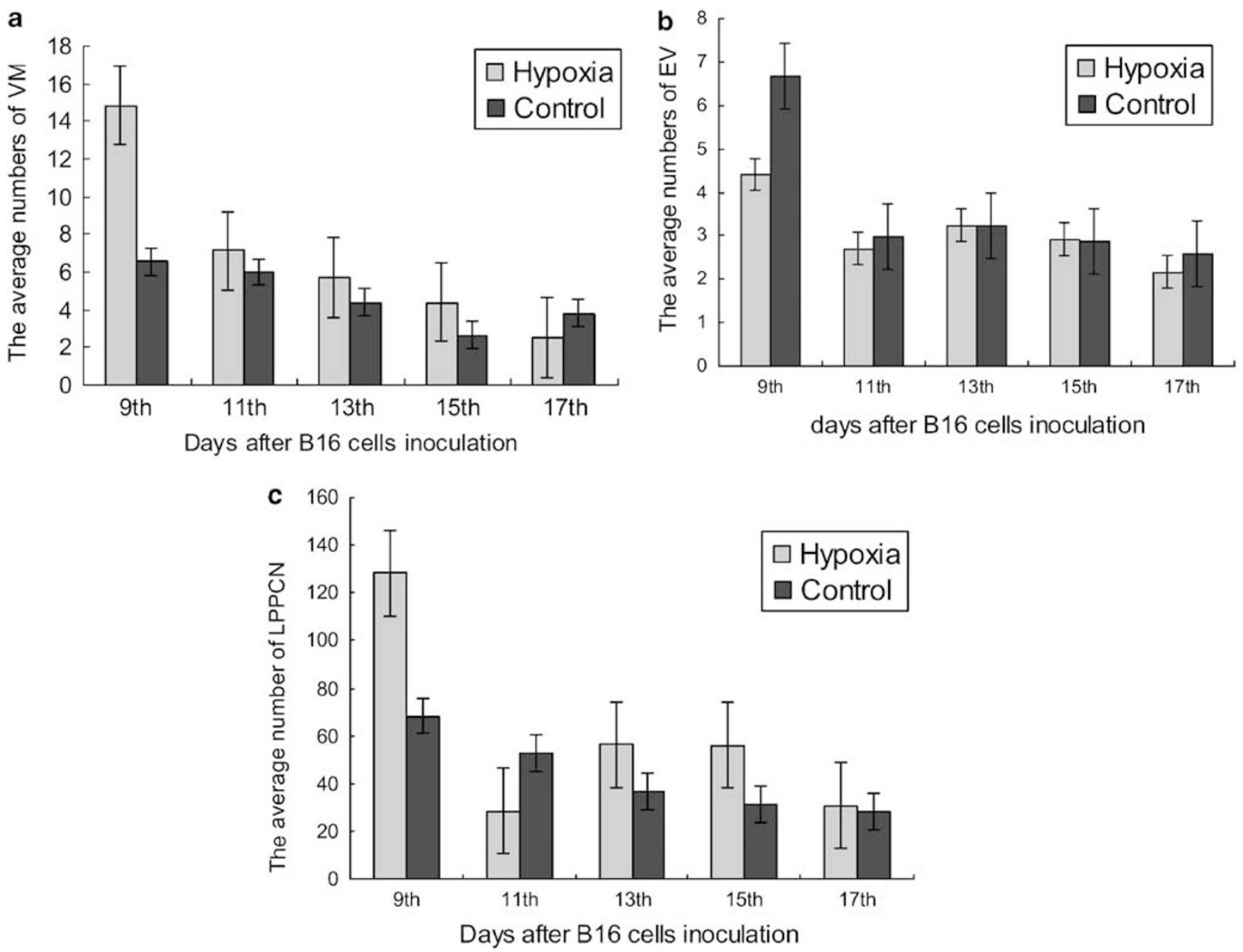

Figure 2 Frequency of VM, EV and LPPCN in different groups. (a) The average number of VM channels in every high power field in the ischemic group and the control group. (b) The average number of EV in every high power field in the ischemic group and the control group. (c) The average number of cells undergoing LPPCN in every high power field in the ischemic group and the control group.

Table 1 Comparison of the count numbers of VM, endothelium-dependent vessels and LPPCN between the ischemic and control groups

$n \quad$ 9th day group $n \quad$ 11th day group $n \quad$ 13th day group $n \quad$ 15th day group $n \quad$ 17th day group

VM

$\begin{array}{llccccccccc}\text { Ischemia } & 9 & 14.85 \pm 9.90^{*} & 7 & 7.12 \pm 3.14 & 9 & 5.70 \pm 3.06 & 5 & 4.40 \pm 2.13 & 9 & 2.54 \pm 0.89 \\ \text { Control } & 9 & 6.54 \pm 3.38 & 7 & 5.99 \pm 2.10 & 9 & 5.53 \pm 3.07 & 5 & 2.64 \pm 2.13 & 9 & 3.80 \pm 2.86\end{array}$

EV

$\begin{array}{lllllllllll}\text { Ischemia } & 9 & 4.41 \pm 0.99 & 7 & 2.70 \pm 1.12 & 9 & 3.24 \pm 2.04 & 5 & 2.92 \pm 1.31 & 9 & 2.17 \pm 0.80 \\ \text { Control } & 9 & 6.66 \pm 4.58 & 7 & 2.99 \pm 2.00 & 9 & 3.22 \pm 1.76 & 5 & 2.88 \pm 1.85 & 9 & 2.58 \pm 1.35\end{array}$

$\angle P P C N$

$\begin{array}{lllllllllll}\text { Ischemia } & 9 & 128.45 \pm 36.32 & 7 & 28.62 \pm 17.32 & 9 & 56.32 \pm 42.28 & 5 & 56.24 \pm 45.73 & 9 & 30.85 \pm 16.74 \\ \text { Control } & 9 & 68.49 \pm 31.30 & 7 & 53.06 \pm 39.59 & 9 & 36.71 \pm 29.23 & 5 & 31.24 \pm 20.01 & 9 & 28.29 \pm 24.24\end{array}$

${ }^{*} P<0.01 ;{ }^{*} P<0.05$. 
showed that the tumor cells undergoing programmed necrosis had nuclei that were negative for TUNEL staining and that there were some apoptotic cells distributed randomly (Figure 3d). Tumor cells with darkly stained nuclei lining the wall of VM and EV were positive for Endo G IHC staining (Figure 3e). Results of ISH showed that LPPCN cells were positive for Endo $G$ mRNA whereas other tumor cells were negative (Figure 3f). Combining the above results in vitro and in vivo, we speculated that Endo G might play an important role in LPPCN progression. LPPCN is also different from traditional necrosis in morphology with IHC staining with dead cells forming a patch. Caspase- 3 IHC staining show that the morphological appearances of typical necrosis display pyknosis, karyorrhexis or karyolysis (features with yellow-brown in Figure 3g).

\section{Electron Microscopy Observation}

Fresh tumor tissues were fixed with glutaraldehyde and fields with linearly patterned programmed necrosis were selected using a light microscope. The areas with programmed necrosis were then observed in more detail with an electron microscope. It was shown that the chromatin often became pyknotic and clumped against the nuclear membrane in cells undergoing programmed necrosis, and red blood cells were found between these cells. The electron micrograph of tumor cells undergoing programmed necrosis showed extensive loss of membrane integrity and limited chromatin condensation. Images also showed that their mitochondria were swollen and had disorganized cristae and the rough endoplasmic reticula were dilated (Figure 3g).

\section{Effects of Hypoxia on Endo G and DNase I mRNA Expression In Vitro}

Melanoma B16 cells were harvested at different time points after exposure to hypoxic conditions. Real-time PCR was performed to detect DNase I and Endo G mRNA expression. As time passed, DNase I expression was elevated in both the hypoxic group and the control group $(P<0.05)$, but the level in the hypoxic group was always higher than that in the control group $(P<0.05$; Figure 4a).

The expression of Endo G mRNA in melanoma cells in the hypoxic group was upregulated and reached its peak at $8 \mathrm{~h}$. It was significantly higher in the hypoxic group than in the control group at 6 and $8 \mathrm{~h}(P<0.05)$. However, there was no longer a significant difference at $24 \mathrm{~h}(P>0.05$; Figure $4 \mathrm{~b})$.

\section{Effects of Hypoxia on Endo G and DNase I mRNA Expression In Vivo}

On the ninth day after inoculation, animals in the ischemic and control groups were killed and melanoma tissues were collected to detect the expression of DNase I and Endo G mRNA. Animal experiment results revealed that Endo G mRNA expression was upregulated markedly in the ischemic group compared with the control group $(P<0.05$; Figure $4 \mathrm{~d})$, and moreover, the expression of Endo G mRNA in melanoma tissues from animal models was remarkably higher than that in cultured cells. By contrast, the DNase I level in the ischemic group of mice was lower than that in the control group $(P<0.05$; Figure $4 \mathrm{c})$. This in vivo result is in direct opposition to the in vitro results; the general expression level in vivo was much lower than that in vitro (Figure 4a and c). These results suggest that, in vivo, hypoxia induced LPPCN in melanoma cells by increasing degradation of DNA by upregulating expression of Endo G.

\section{Survival Analysis of Melanoma Patients with VM and LPPCN}

As the presence of LPPCN and VM might be associated with aggressive cancers, we carefully assessed this issue by correlating the presence of LPPCN and VM with patient survival. Of the 124 cases of melanoma, $41.94 \%$ (52/124) were recognized to have LPPCN and $44.55 \%(54 / 124)$ had VM channels. Kaplan-Meier survival analysis showed the survival time for patients with VM channels was significantly shorter than that of patients without VM channels $(P<0.05)$, with $36.82 \pm 5.64$ months as the average survival time for VMpositive patients and $97.55 \pm 12.19$ months the average survival time for VM-negative patients (Figure 5a). LPPCN had an effect similar to VM on the survival of melanoma patients, and the prognosis of LPPCN-positive cases was worse than that of LPPCN-negative cases. The average survival time of LPPCN-positive and LPPCN-negative cases was $54.13 \pm 10.26$ and $85.52 \pm 11.25$ months, respectively (Figure $5 \mathrm{~b})$. Given the connection between LPPCN and VM, the combined effect on the survival of melanoma patients was assessed. LPPCN and VM double-positive patients had an average survival time of $41.71 \pm 7.75$ months, worse than all other patients grouped together who had an overall survival time of $85.71 \pm 10.62$ months (Figure $5 \mathrm{c}$ ).

\section{DISCUSSION}

In 1999, Maniotis ${ }^{2}$ reported that VM formed by tumor cells instead of endothelial cells appeared in highly aggressive uveal melanomas. Since then, VM has been seen in several malignant tumor types including breast cancer, ${ }^{11}$ liver cancer, ${ }^{12}$ astrocytoma, ${ }^{13}$ ovarian cancer, ${ }^{14}$ prostate cancer $^{15}$ and bidirectional differentiated malignant tumors. ${ }^{16}$ The molecular mechanisms that underlie VM involve the cleavage of laminin via metalloproteinases, VE-cadherin's promotion of adherence of the VM channel wall to tumor cells, tumor cell dedifferentiation and tumor microenvironment. ${ }^{17,18}$ Zhang et al have proposed a three-stage process that begins with VM channels then progresses to mosaic blood vessels and eventually leads to endothelium-dependent blood vessels, ${ }^{10}$ wherein all three patterns participate in tumor blood supply.

The basis for the spatial organization for VM and EV structures is not clear, especially in a high-pressure microenvironment found inside a growing tumor. We have noted the occurrence of some darkly stained tumor cells that seem 

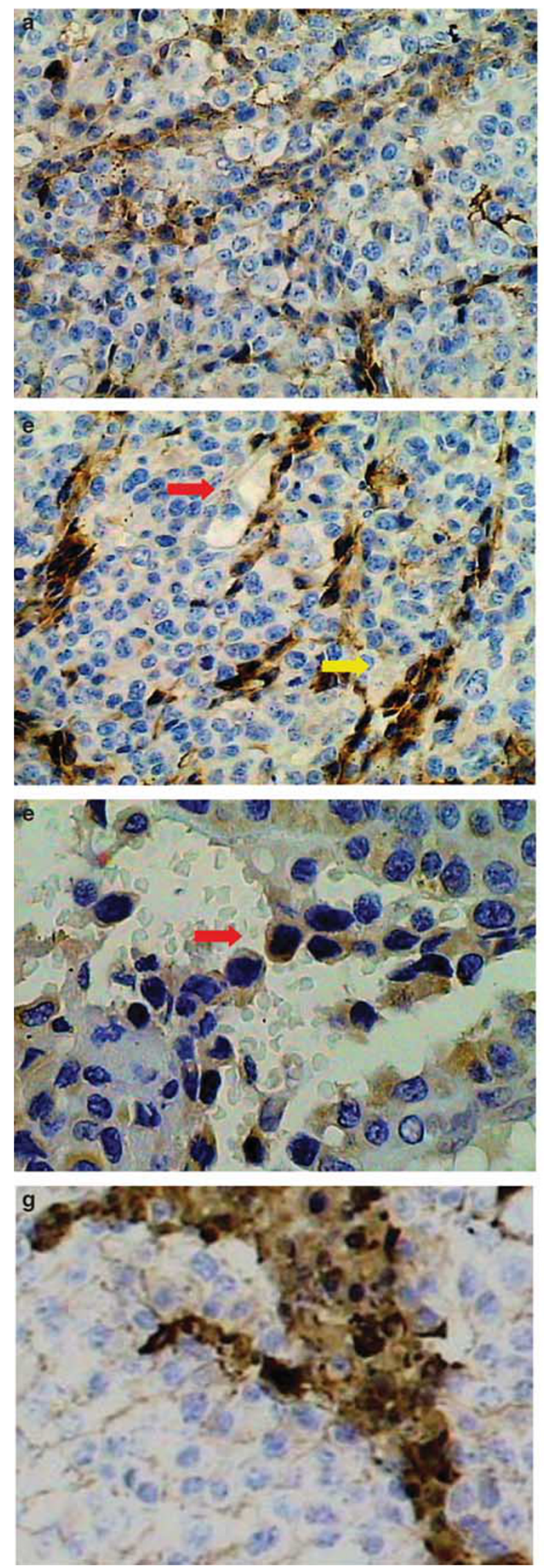

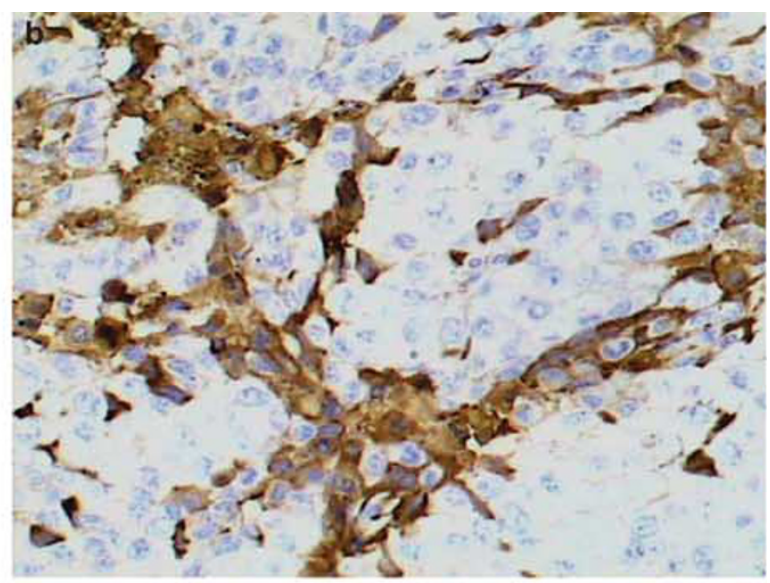

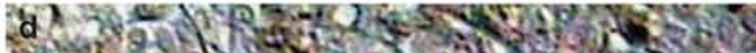

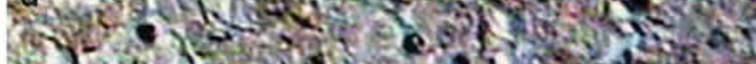

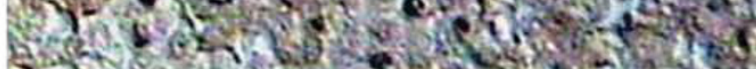

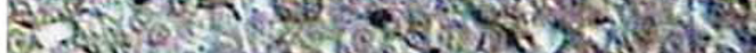

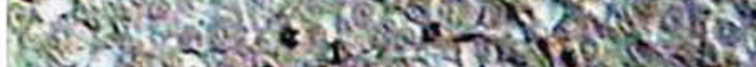
How

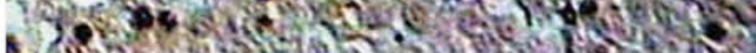
g s A.

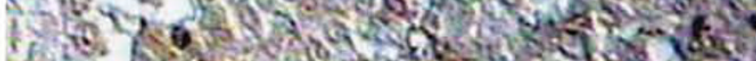

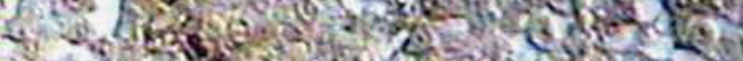

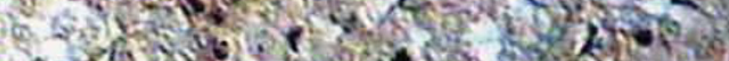

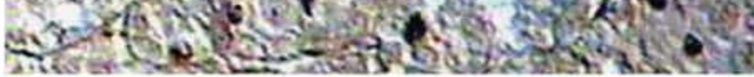
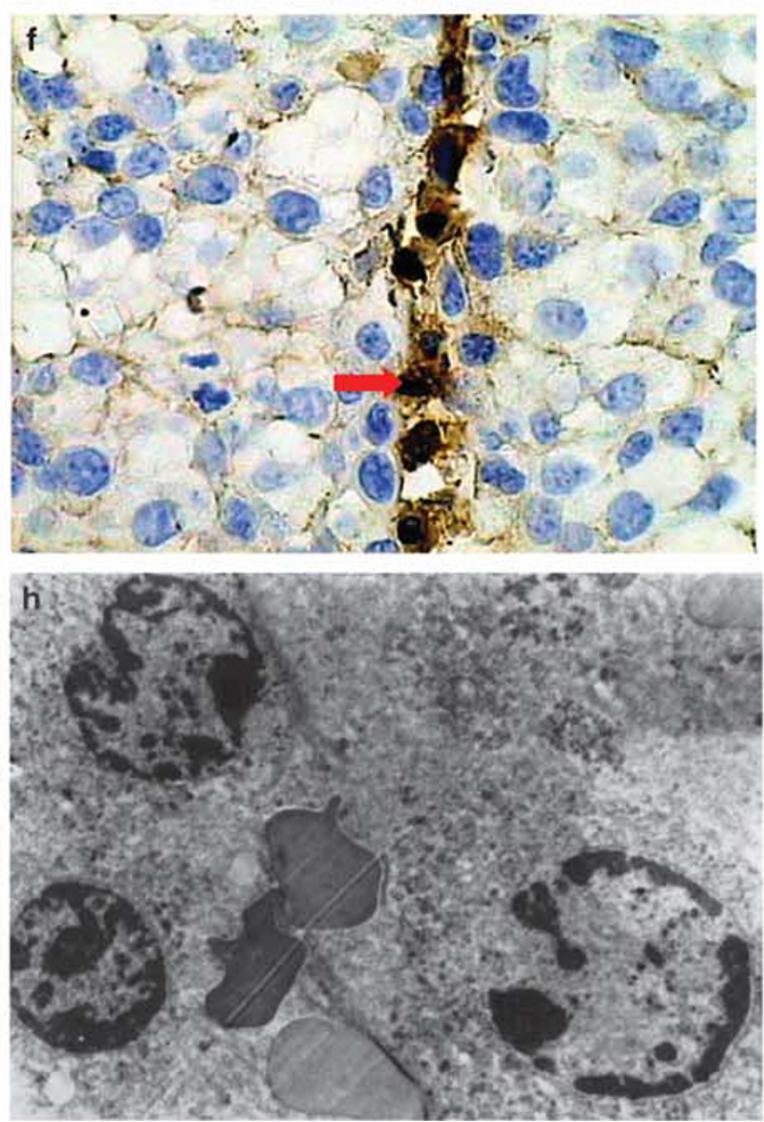
to be distributed in lines and networks that exist in melanoma; we named this phenomenon LPPCN because this necrotic cell death is regulated by apoptosis-related proteins.
In our study, there were more LPPCN cells appearing in the ischemic group. In the control group, the quantity of LPPCN cells was less than it in the ischemic group. Results of our
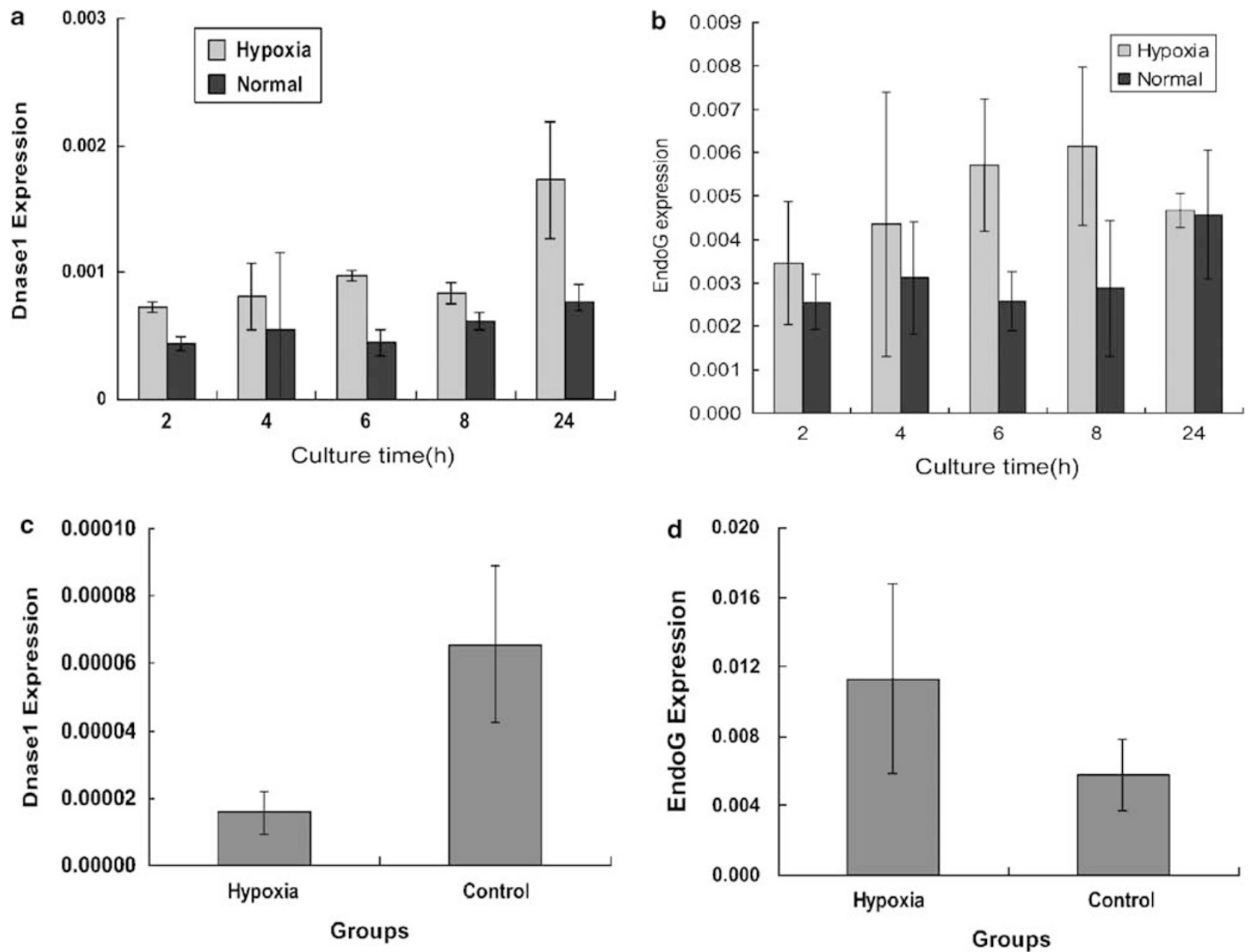

Figure 4 (a-d) Evidence from in vitro and in vivo experiments indicated that hypoxia induced melanoma cells to express increased Endo G, related with hypoxia-induced programmed cell death whereas DNase I mRNA expression was not influenced by hypoxia, especially in vivo, may be associated with tumor cells apoptosis. (a) Comparison of DNase 1 mRNA expression in the hypoxia group and the control group in vitro. DNase I mRNA expression was elevated in both the hypoxic group and the control group, but the level in the hypoxic group was always higher than that in the control group. (b) Comparison of Endo $\mathrm{G}$ mRNA expression in the hypoxia group and the control group in vitro. The expression of Endo $\mathrm{G}$ mRNA in melanoma cells was significantly higher in the hypoxic group than in the control group at 6 and $8 \mathrm{~h}$. (c) Comparison of DNase 1 mRNA expression in the hypoxic group and the control group in vivo. DNase I mRNA expression level in the ischemic group of mice was lower than that in the control group. (d) Comparison of Endo G mRNA expression in the hypoxic group and the control group in vivo. Endo G mRNA expression was upregulated markedly in the ischemic group compared with the control group.

Figure $3(\mathbf{a}-\mathbf{f})$ There were more channels lined with tumor cells that showed darkly stained nuclei in the center of the tumor tissue of the ischemic group. (a) Linearly patterned channels whose inner wall was positive for caspase-3 in the ischemic group, showing the trend of connecting with each other. Caspase-3 IHC staining, SP method, $\times 200$. (b) Linearly patterned channels whose inner wall was positive for caspase-9, showing the trend of connecting with each other in the tumor mass of the ischemic group. Caspase-9 IHC staining, SP method, $\times 200$. (c) Tumor cells in the ischemic group with darkly stained nuclei lining the wall of a VM channel (yellow arrow) and an EV (red arrow) were positive for Bax IHC staining. Bax IHC staining, SP method, $\times 200$. (d) Tumor cells undergoing apoptosis displayed positive TUNEL staining, indicating significant DNA fragmentation, and the nuclei were condensed. They also had a random distribution. The nuclei of the tumor cells undergoing programmed necrosis are negative for TUNEL staining. TUNEL, $\times 200$. (e) Cells undergoing LPPCN lining the wall of a VM channel with red blood cells in it were positive for Endo G IHC staining (red arrow) in the tumor mass of the ischemic group. SP, $\times 100$. (f) ISH for Endo $G$ in melanoma with LPPCN in the ischemic group. LPPCN cells were positive for Endo $G$ mRNA (red arrow). ISH, $\times 400$. (g) Caspase-3 IHC staining for typical necrosis cells distributed in patch and the morphological appearances display pyknosis, karyorrhexis or karyolysis, features with yellow-brown. Caspase-3 IHC staining, SP method, $\times 200$. (h) This electron micrograph shows that tumor cells in the ischemic group with condensed chromatin that are undergoing programmed necrosis form the wall of VM channels. Red blood cells were seen among the necrotic cells $(\times 4000)$. 

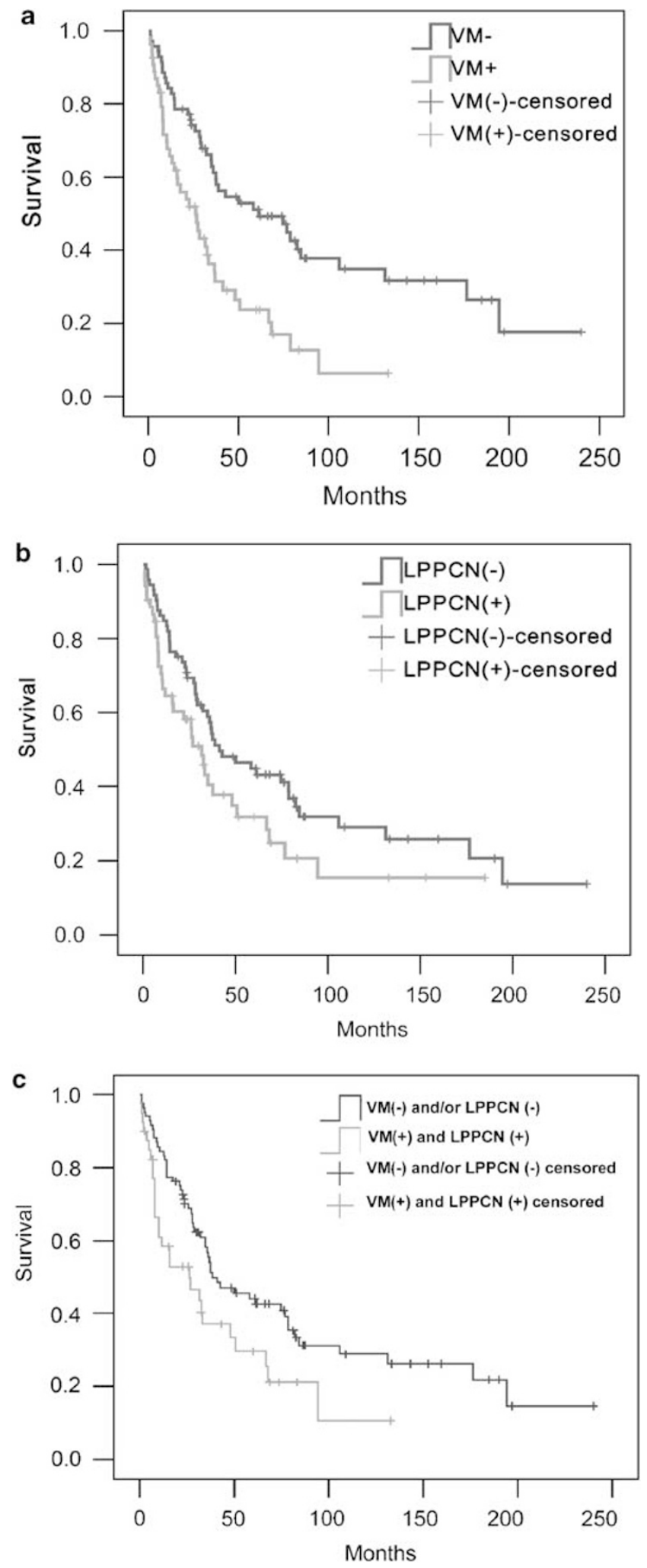

Figure 5 (a and b) Kaplan-Meier survival analysis of melanoma patients based on LPPCN and VM status. (a) VM-positive melanoma patients compared with VM-negative melanoma patients. (b) LPPCN-positive melanoma patients compared with LPPCN-negative melanoma patients. (c) VM and LPPCN double-positive melanoma patients compared with the rest except for $\mathrm{VM}(+) / \mathrm{LPPCN}(+)$ melanoma patients. experiments suggested that ischemia may play an important role in regulating the blood supply formation of melanoma.

To investigate the existence and distribution of these darkly stained cells and the relationship between LPPCN network formation and ischemia, we used a hypoxic animal model bearing melanoma, and killed the animals at regular time points to monitor the development of LPPCN and VM during tumor growth. Results of the animal experiments showed that hypoxia induced some melanoma cells to undergo LPPCN, in which there were remarkably more darkly stained cells in the ischemic group than in the control group, and there were more darkly stained cells present in the early stage of tumor growth than in the late stage. In the early stage of melanoma growth, cells with a darkly stained nucleus appeared in a linear pattern and showed a trend of clustering with each other. IHC and TUNEL staining suggested that these cells undergo programmed necrosis and only appeared in the center of the tumor tissue. As such cell death from LPPCN might provide transient spatial infrastructure for VM and EV development and formation, we hypothesize the following model of vessel progression. In the rapid growth stage characterized by tumor cells hypoxia, blood supplied through EV is not sufficient to satisfy the oxygen and nutrition needs for tumor expansion. This local hypoxic environment induces apoptosis-related genes and genes responsible for LPPCN to be activated. LPPCN development can occur in two different ways. Whether the pattern formed by cells with darkly stained nuclei connects with EV dictates the outcome for these cells. If the LPPCN pattern connects with EV, LPPCN would serve as a spatial infrastructure for VM and EV formation. On the other hand, if the LPPCN does not connect with EV, the darkly stained cells develop into an area of necrosis.

LPPCN is not similar to apoptosis or necrosis. Apoptosis causes characteristic morphologic changes that are genetically regulated and appear in randomly distributed cells. It has generally been believed that necrosis is a passive process, during which nuclear changes appear in the forms of karyolysis, pyknosis and karyorrhexis. ${ }^{19}$ The traditional view has held that necrosis is pathologic and a passive death, whereas apoptosis is programmed cell death, considered a physiological, active death. It has become increasingly clear that the two cell death phenotypes and molecular mechanisms are highly diverse. ${ }^{20,21}$ Programmed necrosis is a phenomenon that has been brought forward by Zong $e t a l^{5}$ and Junying Yuan et al. ${ }^{6}$ Programmed necrosis is a newly characterized type of cell death in ischemic brain injury animal models. Morphologically, programmed necrosis displays pyknosis, karyorrhexis or karyolysis, features that are different from the apoptotic bodies and DNA fragmentation typical of apoptosis. ${ }^{22}$ Apoptotic cells distribute randomly whereas the distribution of cells undergoing programmed necrosis is regulated by the tumor cells themselves. ${ }^{23,24}$ Furthermore, LPPCN is also different from traditional necrosis in morphology, with dead cells forming a line shape as opposed to a 
patch that is most commonly observed in traditional necrotic death in most tissues.

According to the results of IHC staining, we speculated that the molecular mechanism underling LPPCN might be similar to the mitochondria-dependent apoptosis-signaling pathway. However, the distinct morphology between these two death patterns might be due to the difference in particular endonucleases involved in each type. DNase I, a Ca ${ }^{+2}$ and $\mathrm{Mg}^{2+} / \mathrm{Mn}^{2+}$-dependent endonuclease with optimal performance at $\mathrm{pH}$ 7.5, hydrolyzes double-stranded DNA by introducing single strand nicks that generate $5^{\prime}$-phospho-triand/or -tetra-oligonucleotides. ${ }^{25}$ DNase I targets the binding site between DNA and the nucleosome, hence, the DNA fragments cleaved by DNase I are $180 \mathrm{bp}$ and multiples thereof. Markus Napirei et al reported that DNase I not only mainly mediates the chromatin breakdown in apoptosis, but also partly participates in the chromatin breakdown of necrotic cells in vitro. This in vitro effect is achieved through diffusion from the extracellular fluid through the ruptured plasma membrane into the cytoplasm and nucleus of necrotic cells. Endo $G$ is the most active endonuclease in eukaryotic cells, and the gene encoding it is conserved from yeast to human beings. ${ }^{26}$ Endo $G$ is nonspecific and can hydrolyze double-stranded DNA, single-stranded DNA and RNA with peak performance at $\mathrm{pH} 7.0$ in a $\mathrm{Mg}^{2+}$-dependent manner. Endo $G$ in the mitochondrial intermembrane space has multiple functions. It prevents degradation of genomic DNA in normal cells, but is released from mitochondria in response to apoptotic signals after activation. ${ }^{27,28}$ Endo G is an essential member of the mitochondria-dependent apoptosis signaling pathway. Proapoptotic Bcl-2 family members such as Bid and Bim induce mitochondrial outer-membrane permeabilization resulting in the release of Endo G. The Endo G and AIF (apoptosis inducing factor) complex is then transmitted into the nucleus to degrade DNA. It has been shown that the activation of caspase 3 can induce Endo G release, but Endo $G$ is normally associated with the 'caspaseindependent' apoptotic pathways. ${ }^{29,30}$ Damage to the mitochondrial outer membrane leads to an Endo G-related programmed cell death, even with inactivation of the caspase family. In this study, we found that Endo G may be associated with hypoxia-induced programmed cell death in melanoma cells. Evidence from in vitro and in vivo experiments indicated that hypoxia induced melanoma cells to express increased Endo G, whereas DNase I mRNA expression was not influenced by hypoxia, may be associated with tumor cells apoptosis. Results of Endo G IHC staining and mRNA ISH confirmed the expression of Endo G protein and mRNA in LPPCN cells. Some LPPCN cells lining the walls of VM channels were positive for Endo G IHC staining. These findings suggest that Endo $G$ was critical for LPPCN formation of melanoma cells under hypoxic conditions. ${ }^{31}$

Programmed necrosis is regulated by apoptosis-related genes and is associated with signal transducers such as protein kinases and phosphatases. ${ }^{32}$ Vartanian et $a l^{33}$ have recently shown that the formation of capillary-like structures (VM) requires apoptotic cell death through activation of a caspase-3-dependent mechanism. They have shown that the caspase inhibitor zVAD-fmk and a caspase- 3 inhibitor DEVD blocked capillary-like structure formation in an in vitro assay, supporting a mechanism based on the induction of apoptosis followed by an altered form of cell death without nuclear fragmentation resembling secondary necrosis. Mitochondrial disorder caused by hypoxia or energy failure can trigger programmed necrosis cascades that involve the bax family of genes. ${ }^{34}$ Hypoxia-induced mitochondrial dysfunction, which causes release of cytochrome $\mathrm{C}$ and consequently procaspase expression, seems to be the initial event of programmed necrosis. ${ }^{35,36}$

The unique structure of VM channels facilitates the hematogeneous metastasis of tumor cells. Tumor cells lining the inner surface of VM channels are directly exposed to blood flow, and the tumor cells that leak out would migrate through the blood stream and metastasize to other organs. In fact our studies have demonstrated that LPPCN and VM are associated with poor clinical prognosis in melanoma patients, especially in the cases that display both features. There were more LPPCN and VM in tumor mass which melanoma cells had poorer differentiation and faster growth. The growth rate was associated with the degree of hypoxia. In the melanoma patients with poorer differentiation, tumor tissues with rapid growth and serious hypoxia lead to the poor prognosis.

In this study, we propose a special death pattern for tumor cells and suggest that this pattern might be associated with $\mathrm{VM}$ and tumor angiogenesis and hypoxia may be an important regulating factor. LPPCN is not exclusive to melanoma but exists in many kinds of high-grade malignant tumors. However, its unique appearance was often ignored by pathologists. Based on our observations and investigation, we begin describing this interesting phenomenon and try to provide a model of vessel development during tumor progression, in which a local hypoxic environment generated during the early stage of tumor growth induces LPPCN which may serve as the spatial foundation for further blood vessel development, ie, angiogenesis. However, the profound molecular mechanisms involved in LPPCN and the relationship between the LPPCN network and tumor blood supply still remain unclear and need further biochemical and molecular studies. For instance, with the exception of Endo G and DNase I, other endonucleases might participate in the cleavage of DNA during LPPCN and the presence of these other endonucleases deserves further study. Here we have focused on the difference in Endo G and DNase 1 protein expression under hypoxic conditions and showed that Endo G, but not DNase I, might play a role in LPPCN in vivo.

\section{ACKNOWLEDGEMENTS}

We thank Valerie Dunmire for her expert editorial assistance with this article. This work was partially supported by the National Science Foundation of China (30770828) and the Key National Science Foundation of China (30830049). 
1. Seftor EA, Meltzer PS, Kirschmann DA, et al. Molecular determinants of human uveal melanoma invasion and metastasis. Clin Exp Metastasis 2002;19:233-246.

2. Maniotis AJ, Folberg R, Hess A, et al. Vascular channel formation by human melanoma cells in vivo and vitro: vasculogenic mimicry. Am J Pathol 1999;155:739-752.

3. Hendrix MJ, Seftor EA, Meltzer PS, et al. Expression and functional significance of VE-cadherin in aggressive human melanoma cells: role in vasculogenic mimicry. Proc Natl Acad Sci USA 2001;98:8018-8023.

4. Sun B, Zhang D, Zhang S, et al. Hypoxia influences vasculogenic mimicry channel formation and tumor invasion-related protein expression in melanoma. Cancer Lett 2007;249:188-197.

5. Zong WX, Ditsworth D, Bauer DE, et al. Alkylating DNA damage stimulates a regulated form of necrotic cell death. Genes Dev 2004;18:1272-1282.

6. Yuan J, Lipinski $M$, Degterev A. Diversity in the mechanisms of neuronal cell death. Neuron 2003;40:401-413.

7. Yakolev AG, Faden Al. Mechanisms of neural cell death: implications for development of neuroprotective treatment strategies. NeuroRx 2004; 1:5-16.

8. Zhang S, Zhang D, Sun B. Vasculogenic mimicry: current status and future prospects. Cancer Lett 2007;254:157-164.

9. Xu XC, Ro JY, Lee JS, et al. Differential expression of nuclear retinoid receptors in normal, premalignant, and malignant head and neck tissues. Cancer Res 1994;54:3580-3587.

10. Zhang $S$, Zhang D, Wang $Y$, et al. Morphologic research of microcirculation pattern in human and animal melanoma. Med Oncol 2006;23:403-409.

11. Shirakawa K, Kobayashi H, Sobajima J, et al. Inflammatory breast cancer: vasculogenic mimicry and its hemodynamics in an inflammatory breast cancer xenograft model. Breast Cancer Res 2003;5:136-139.

12. Sun $B$, Zhang $S$, Zhang $D$, et al. Vasculogenic mimicry is associated with high tumor grade, invasion and metastasis, and short survival in patients with hepatocellular carcinoma. Oncol Rep 2006;16:693-698.

13. Yue WY, Chen ZP. Does vasculogenic mimicry exist in astrocytoma? J Histochem Cytochem 2005;53:997-1002.

14. Sood AK, Fletcher MS, Coffin JE, et al. Functional role of matrix metalloproteinases in ovarian tumor cell plasticity. Am J Obstet Gynecol 2004;190:899-909.

15. Sharma N, Seftor RE, Seftor EA, et al. Prostatic tumor cell plasticity involves cooperative interactions of distinct phenotypic subpopulations: role in vasculogenic mimicry. Prostate 2002;50: 189-201

16. Sun B, Zhang S, Zhao X, et al. Vasculogenic mimicry is associated with poor survival in patients with mesothelial sarcomas and alveolar rhabdomyosarcomas. Int J Oncol 2004;25:1609-1614.

17. Hendrix MJ, Seftor EA, Kirschmann DA, et al. Remodeling of the microenvironment by aggressive melanoma tumor cells. Ann NY Acad Sci 2003;995:151-161.

18. Folkman J. Role of angiogenesis in tumor growth and metastasis. Semin Oncol 2002;19:15-18.

19. Assuncao Guimaraes C, Linden R. Programmed cell deaths. Apoptosis and alternative deathstyles. Eur J Biochem 2004;271:1638-1650.
20. Degterev A, Boyce M, Yuan J. The channel of death. J Cell Biol 2001;155:695-698.

21. Lin Y, Choksi S, Shen HM, et al. Tumor necrosis factor-induced nonapoptotic cell death requires receptor-interacting proteinmediated cellular reactive oxygen species accumulation. J Biol Chem 2004;279:10822-10828.

22. Degterev $A$, Lugovskoy $A$, Cardone $M$, et al. Identification of smallmolecule inhibitors of interaction between the $\mathrm{BH} 3$ domain and $\mathrm{BCl}-\mathrm{xL}$. Nat Cell Biol 2001;3:173-182.

23. Proskuryakov SY, Gabai VL, Konoplyannikov AG. Necrosis is an active and controlled form of programmed cell death. Biochemistry (Mosc) 2002;67:387-408.

24. Lugovskoy AA, Degterev Al, Fahmy AF, et al. A novel approach for characterizing protein ligand complexes: molecular basis for specificity of small-molecule $\mathrm{Bcl}-2$ inhibitors. J Am Chem Soc 2002;124:1234-1240.

25. Li LY, Luo X, Wang X. Endonuclease G is an apoptotic DNase when released from mitochondria. Nature 2001;412:95-99.

26. Widlak P, Li LY, Wang X, et al. Action of recombinant human apoptotic endonuclease $\mathrm{G}$ on naked DNA and chromatin substrates: cooperation with exonuclease and DNase I. J Biol Chem 2001;276:48404-48409.

27. Strauss G, Westhoff MA, Fischer-Posovszky P, et al. 4-hydroperoxycyclophosphamide mediates caspase-independent T-cell apoptosis involving oxidative stress-induced nuclear relocation of mitochondrial apoptogenic factors AIF and Endo G. Cell Death Differ 2008;15: 332-343.

28. Kim JS, Lee JH, Jeong WW, et al. Reactive oxygen species-dependent Endo $\mathrm{G}$ release mediates cisplatin-induced caspase-independent apoptosis in human head and neck squamous carcinoma cells. Int J Cancer 2008;122:672-680.

29. Dupont-Versteegden EE, Strotman BA, Gurley CM, et al. Nuclear translocation of Endo $G$ at the initiation of disuse muscle atrophy and apoptosis is specific to myonuclei[J]. Am J Physiol Regul Integr Comp Physiol 2006;291:R1730-R1740.

30. David KK, Sasaki M, Yu SW, et al. Endo G is dispensable in embryogenesis and apoptosis. Cell Death Differ 2006;13:1147-1155.

31. Liu PL, Chen YL, Chen YH, et al. Wood smoke extract induces oxidative stress-mediated caspase-independent apoptosis in human lung endothelial cells: role of AIF and Endo G. Am J Physiol Lung Cell Mol Physiol 2005;289:L739-L749.

32. Degenhardt K, Sundararajan R, Lindsten T, et al. Bax and Bak independently promote cytochrome $\mathrm{C}$ release from mitochondria. J Biol Chem 2002;277:14127-14134.

33. Vartanian AA, Burova OS, Stepanova EV, et al. The involvement of apoptosis in melanoma vasculogenic mimicry. Melanoma Res 2007;17:1-8.

34. Ghobrial IM, Witzig TE, Adjei AA. Targeting apoptosis pathways in cancer therapy. CA Cancer J Clin 2005;55:178-194.

35. Chan FK, Shisler J, Bixby JG, et al. A role for tumor necrosis factor receptor-2 and receptor-interacting protein in programmed necrosis and antiviral responses. J Biol Chem 2003;278:51613-51621.

36. Weber GF, Menko AS. The canonical intrinsic mitochondrial death pathway has a non-apoptotic role in signaling lens cell differentiation. J Biol Chem 2005;280:22135-221345. 